\title{
A C/X Dual-band Wide-angle Reflective Polarization Rotation Metasurface
}

\author{
Yulong ZHOU, Xiangyu CAO, Jun GAO, Sijia LI \\ Information and Navigation College, Air Force Engineering University, Xi' an 710077, China \\ qq412852512@126.com, xiangyucaokdy@163.com,gjgj9694@163.com,1sj051@126.com \\ Submitted December 22, 2016 / Accepted March 27, 2017
}

\begin{abstract}
In this paper, a $C / X$ dual-band wide-angle reflective polarization rotation metasurface (PRMS) with high rotation efficiency is proposed and realized. Aiming to miniaturize the size of the unit cell, a metallic flower-like shape ring is selected to extend the current path and the 45 degree slanting stitch along diagonal direction is used to form the asymmetric structure. The simulated results show that the proposed PRMS achieves polarization rotation at 4.61 GHz and $8.67 \mathrm{GHz}$ with high efficiency, at which the linear polarization incident wave is converted into its orthogonal polarization after reflection. Furthermore, the high polarization rotation efficiency of the proposed PRMS is maintained under an oblique incident direction from $0^{\circ}$ to $60^{\circ}$. To verify the simulated results, the proposed PRMS is fabricated and measured. The measured results are in good accordance with the simulated ones.
\end{abstract}

\section{Keywords}

Polarization rotation metasurface, dual band, wide angle

\section{Introduction}

As polarization is one of the most important characteristics of the electromagnetic (EM) waves, it is of great significance to be manipulated. Optical activity crystals and Faraday effects are traditionally used to realize the polarization rotation, whereas these methods usually require quite long propagation distance to get the phase accumulation resulting in bulky devices. Therefore, novel rotation devices with small thickness and light weight need to be developed.

Metasurface, as a two-dimensional artificial material, has received more and more interest for its novel electronic properties to realize cloaks [1], [2], absorbers [3-5], and polarization convertors [6-9]. With small thickness and light weight, the polarization rotation metasurface (PRMS) can realize polarization rotation in multi-band or broadband. Based on the way of controlling the polarization states, the PRMS can be divided into transmitted and reflective PRMS. For the transmitted PRMS, planar or 3D chiral metasurface have been proposed to control the polarization [10-12]. For the reflective PRMS, anisotropic metasurface with single- or multi-layer is employed to realize broadband PRMS [13-15]. Moreover, many structures are designed for better performance, such as V-shaped patches [14], U-shaped patches [16], and square patch loaded with metallic holes along the diagonal direction [13]. However, the polarization rotation efficiency of the PRMS under different incident directions is less discussed in previous articles. For wideband or multiband applications [16-22], the angle stability of the PRMS is still one of the research interests.

In this paper, a $\mathrm{C} / \mathrm{X}$ dual-band wide-angle reflective PRMS with high polarization rotation efficiency is proposed. A flower-like shape ring with a 45 degree slanting stitch along diagonal direction is designed to form the anisotropy structure. The current path of the flower-like shape ring is larger, thus the size of the unit cell is smaller. The proposed PRMS can realize nearly $100 \%$ polarization rotation efficiency at $4.61 \mathrm{GHz}$ and $8.67 \mathrm{GHz}$. Furthermore, the proposed PRMS can remain high polarization rotation efficiency under different incident directions from $0^{\circ}$ to $60^{\circ}$. When the incident angle comes to $60^{\circ}$, the magnitude of PCR is still more than $79 \%$ at the resonant frequencies. The performance of the PRMS is analyzed by induced surface current distribution, physical theory and measurement results.

\section{Design and Analysis}

\subsection{Design Principles}

The diagram of the proposed units is shown in Fig. 1. The PRMS is etched on a substrate with period of $10.4 \mathrm{~mm}$, thickness of $2.0 \mathrm{~mm}$, dielectric constant of 4.4 and loss tangent of 0.001 . A flower-like shape ring with a 45 degree slanting stitch along diagonal direction is on the top of the substrate and a full metallic plate is at the bottom.

As depicted in Fig. 1(b), the flower-like shape ring is a combination of four three-quarter metallic rings in a consecutive implementation. The current path of the flowerlike shape ring is larger, thus the size of the unit cell is 


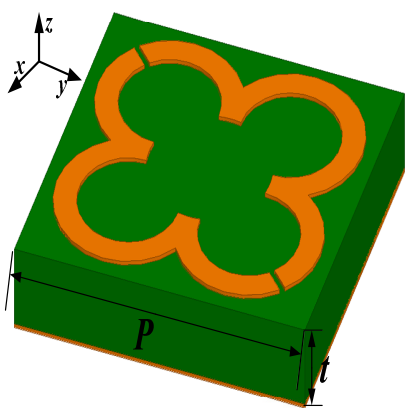

(a)

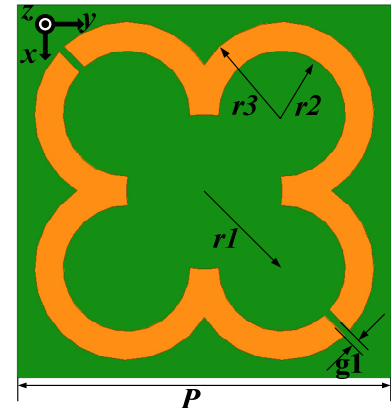

(b)
Fig. 1. The unit cell of the proposed PRMS: (a) Topological view; (b) Front view. The corresponding geometrical parameters are designed as: $P=10.4 \mathrm{~mm}, \quad r 1=$ $3.05 \mathrm{~mm}, r 2=1.75 \mathrm{~mm}, r 3=2.55 \mathrm{~mm}, g 1=0.2 \mathrm{~mm}$ and $t=2 \mathrm{~mm}$.

smaller. The 45 degree slanting stitch along diagonal direction is used to form asymmetric structure, which can convert the linear polarization incident wave into its orthogonal polarization after reflection. Thus, the dualband polarization rotation is achieved through a miniaturized structure. The geometrical parameters are also given in Fig. 1.

\subsection{Numerical Simulations}

In order to verify the designed dual-band PRMS, numerical simulations were performed by using the commercial software HFSS. Periodic boundary condition and Floquet ports are used in HFSS. The metallic material is copper and the substrate is FR4. Owing to the anisotropy of the unit cell structure, the reflected wave generally consists of both co- and cross-polarized components. Resulting from a full metallic plate backed, the energy of the transmitted wave is zero, and the energy of the incident wave is all reflected ignoring the energy loss in the substrate.

For a y-polarized incident wave, the co- and crosspolarized components are defined as

$$
\begin{aligned}
& r_{\text {yy }}=\left|\boldsymbol{E}_{\mathrm{ry}}\right| /\left|\boldsymbol{E}_{\mathrm{iy}}\right|, \\
& r_{\mathrm{yx}}=\left|\boldsymbol{E}_{\mathrm{rx}}\right| /\left|\boldsymbol{E}_{\mathrm{iy}}\right| .
\end{aligned}
$$

What's more, the polarization conversion ratio $P C R$ is written as

$$
P C R=r_{\mathrm{yx}}^{2} /\left(r_{\mathrm{yx}}^{2}+r_{\mathrm{xx}}^{2}\right) .
$$

To understand the polarization state of the reflective wave, the azimuth angle $\psi$ and ellipticity angle $\kappa$ are introduced and expressed as follows:

$$
\begin{aligned}
& \tan (2 \psi)=\frac{2 r_{\mathrm{yx}} \cos (\zeta)}{1-r_{\mathrm{yx}}^{2}}, \\
& \sin (2 \kappa)=\frac{2 r_{\mathrm{yx}} \cos (\zeta)}{1+r_{\mathrm{yx}}^{2}}, \\
& \zeta=\text { Phase }_{r_{\mathrm{yx}}}-\text { Phase }_{r_{\mathrm{yy}}} .
\end{aligned}
$$

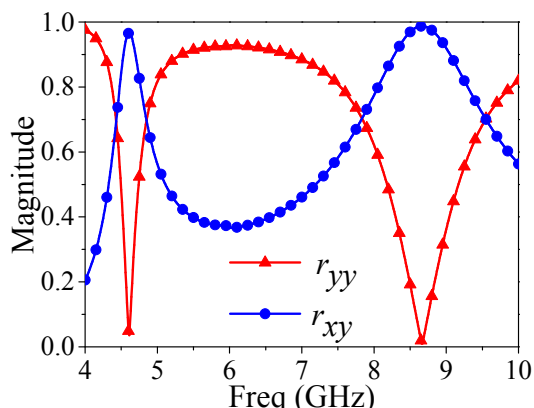

(a)

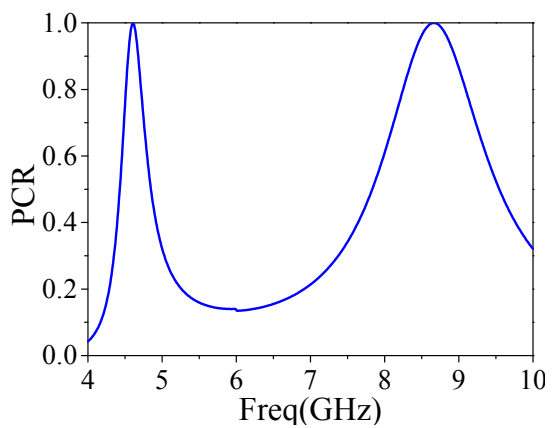

(b)

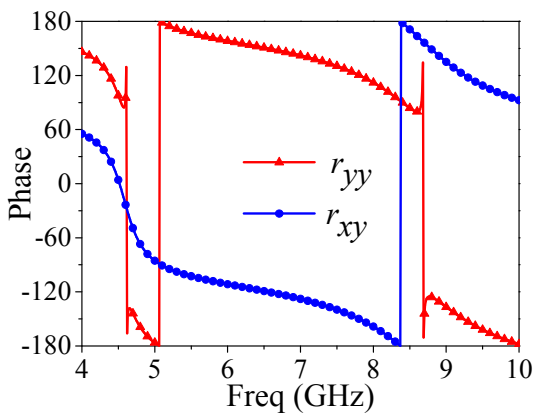

(c)

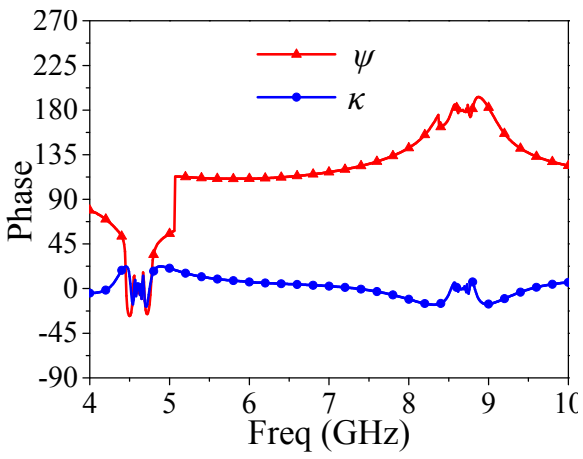

(d)

Fig. 2. The reflection coefficient of the proposed PRMS: (a) the magnitude of $r_{\mathrm{xy}}$ and $r_{\mathrm{yy}}$; (b) PCR; (c) the phase of $r_{\mathrm{xy}}$ and $r_{\mathrm{yy}}$; (d) the azimuth angle $\psi$ and ellipticity angle $\kappa$.

The simulated reflection results of the PRMS are shown in Fig. 2. Due to the less loss tangent of the dielectric substrate, the magnitude of $r_{\mathrm{xy}}$ is larger than 0.95 at 4.61 GHz and $8.67 \mathrm{GHz}$, at which the PCR is nearly $100 \%$, shown in Fig. 2(a) and Fig. 2(b). It means that nearly all energy of y-polarized incident wave is converted into the $\mathrm{x}$-polarized reflected one at these two key frequencies respectively. As depicted in Fig. 2(d), the azimuth angle $\psi$ 


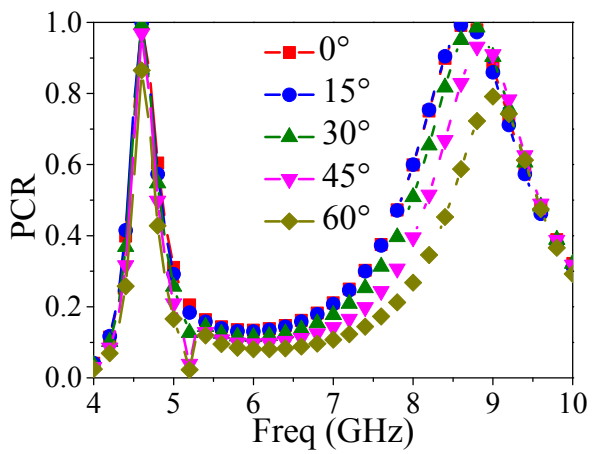

Fig. 3. The angle stability of the proposed PRMS.

\begin{tabular}{|c|c|c|c|}
\hline $\begin{array}{c}\text { Cross } \\
\text { polarization } \\
\text { convertor }\end{array}$ & $\begin{array}{c}\text { Resonance } \\
\text { number }\end{array}$ & $\begin{array}{c}\text { Angle } \\
\text { stability }\end{array}$ & Period \\
\hline$[19]$ & 3 & $\begin{array}{c}\text { Not } \\
\text { mentioned }\end{array}$ & $0.43 \lambda_{0}$ \\
\hline$[21]$ & 2 & $45 \mathrm{deg}$ & $0.30 \lambda_{0}$ \\
\hline Our work & 2 & $60 \mathrm{deg}$ & $0.16 \lambda_{0}$ \\
\hline
\end{tabular}

Tab. 1. Performance comparison.

is nearly $0^{\circ}$ at $4.61 \mathrm{GHz}$ and $180^{\circ}$ at $8.67 \mathrm{GHz}$. In addition, the ellipticity angle $\kappa$ is approximately $2^{\circ}$ at $4.61 \mathrm{GHz}$ and $-0.6^{\circ}$ at $8.67 \mathrm{GHz}$, which indicates that the linear polarization incident wave can be converted into its orthogonal polarization after reflection.

The angle stability of the proposed PRMS is also investigated. The azimuth is defined by angle $\theta$, indicating the azimuth between the incidence wave vector and PRMS surface vector. As the angle $\theta$ changing from $0^{\circ}$ to $60^{\circ}$, the resonant frequencies and the PCR magnitude of the proposed PRMS are shown in Fig. 3. With the angle $\theta$ increasing, the first resonant frequency remains unchanged, and the second resonant frequency is slightly shifted to a higher frequency. Moreover, the magnitude of PCR at resonant frequencies is keeping unchanged or decreasing slightly, when the incident angle increases to $45^{\circ}$. What's more, when the incident angle comes to $60^{\circ}$, the magnitude of PCR is still coming to $86.5 \%$ at the first resonant frequency, and $79.1 \%$ at the second resonant frequency. The proposed PRMS possesses good angle stability.

Table 1 lists the performance comparison between three cross polarization convertors. Compared with the other two convertors, the proposed convertor has a smaller period and better angle stability at the same time. The overall length of the flower-like shape ring contains four continuously three-quarter circles, which is larger than previous literatures. Therefore, the size of the unit cell is smaller, only $0.16 \lambda_{0} \times 0.16 \lambda_{0}\left(\lambda_{0}\right.$ represents free space wavelength at the minimal resonant frequency in the bandwidth). Moreover, the proposed PRMS is better in angle stability. The PCR at the second resonance frequency is near $80 \%$, when the incident angle comes to $60^{\circ}$.

\subsection{Physical and Theoretical Discussion}

In order to get a physical insight into these plasmon frequencies, the surface current distribution on the flower- like shape ring and the ground are depicted in Fig. 4. As demonstrated in Fig. 4, the overall direction of the surface current on the flower-like shape ring is antiparallel to those on the metallic ground presenting equivalent current loop. Then, the magnetic dipole is forming [23]. Moreover, the difference in the surface current directions indicates that the different magnetic responses are the results of the multi-order resonance modes.

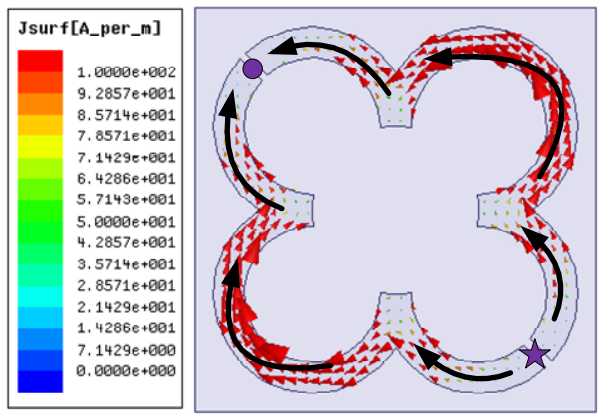

(a)

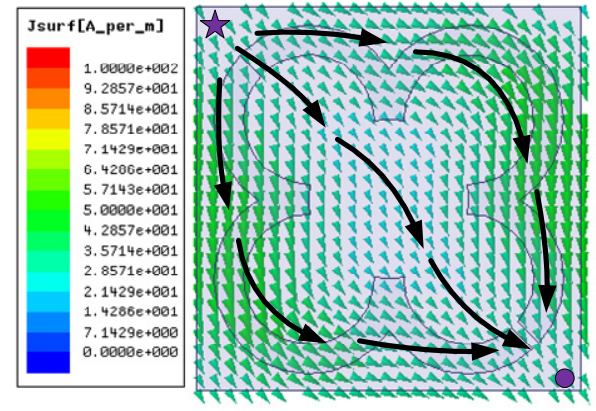

(b)

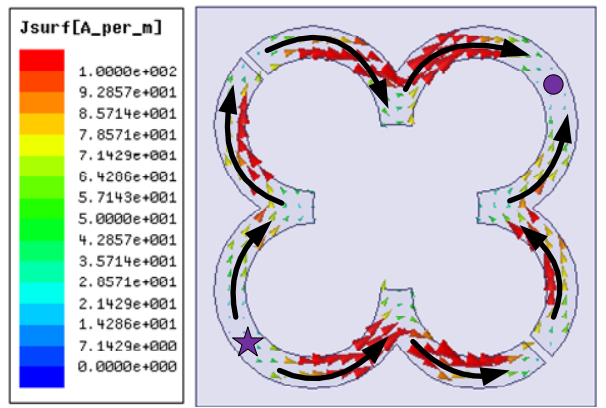

(c)

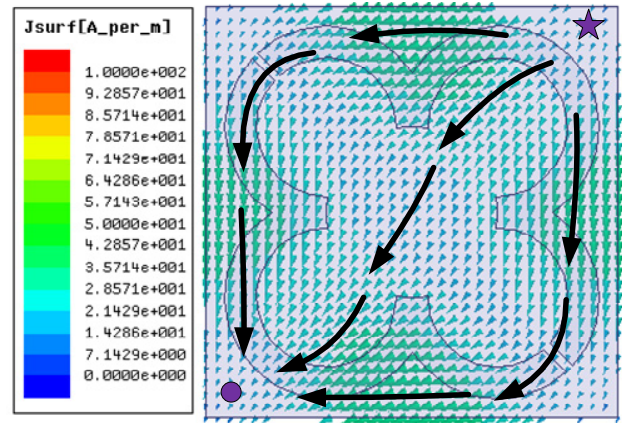

(d)

Fig. 4. The surface current distribution of the PRMS: (a) Top surface current at $4.61 \mathrm{GHz}$; (b) Bottom surface current at $4.61 \mathrm{GHz}$; (c) Top surface current at $8.67 \mathrm{GHz}$; (d) Bottom surface current at $8.67 \mathrm{GHz}$. 
To understand the response of the proposed PRMS to the y-polarized incident EM wave theoretically, we decompose the y-polarized incident wave into two orthogonal axis $\mathrm{u}$ and $v$, which are along the 45 degree direction with respect to axis $x$ and axis y shown in Fig. 5. Hence, the ypolarized incident wave can be written as (7):

$$
\boldsymbol{E}_{\text {iy }}=\left|\boldsymbol{E}_{\mathrm{iy}}\right| \boldsymbol{e}_{\mathrm{y}}=E_{\mathrm{iu}} \boldsymbol{e}_{\mathrm{u}}+E_{\mathrm{iv}} \boldsymbol{e}_{\mathrm{v}} .
$$

Meanwhile, the reflective wave can be defined as (8):

$$
\boldsymbol{E}_{\mathrm{ry}}=\left|\boldsymbol{E}_{\mathrm{iy}}\right| \boldsymbol{e}_{\mathrm{y}}=r_{\mathrm{u}} E_{\mathrm{iu}} \boldsymbol{e}_{\mathrm{u}}+r_{\mathrm{v}} E_{\mathrm{iv}} \boldsymbol{e}_{\mathrm{v}} .
$$

Moreover, $\Delta \Phi$ is used to define the phase difference between $r_{\mathrm{u}}$ and $r_{\mathrm{v}}$, and then the relationship between $r_{\mathrm{u}}$ and $r_{\mathrm{v}}$ can be written as (9):

$$
r_{\mathrm{v}}=r_{\mathrm{u}} \exp (-\mathrm{j} \Delta \Phi) .
$$

Thus, the reflective wave $\boldsymbol{E}_{\mathrm{r}}$ can be modified by inserting (9) into (8):

$$
\boldsymbol{E}_{\mathrm{r}}=r_{\mathrm{u}} E_{\mathrm{iu}} \boldsymbol{e}_{\mathrm{u}}+r_{\mathrm{u}} \exp (-\mathrm{j} \Delta \Phi) E_{\mathrm{iv}} \boldsymbol{e}_{\mathrm{v}} .
$$

Due to the less tangent loss substrate and the anisotropy of the unit cell, the magnitude of $r_{\mathrm{u}}$ and $r_{\mathrm{v}}$ would almost be equal to 1, demonstrated in Fig. 6(a). When it comes to the resonant frequencies, the $\Delta \Phi$ is nearly $180^{\circ}$, depicted in Fig. 6(b). By again looking to (10) and setting $r_{\mathrm{u}}=r_{\mathrm{v}} \approx 1$, and $\Delta \Phi \approx 180^{\circ}$, Equation (10) is equal to

$$
\begin{aligned}
\boldsymbol{E}_{\mathrm{r}} & =r_{\mathrm{u}} E_{\mathrm{iu}} \boldsymbol{e}_{\mathrm{u}}+r_{\mathrm{u}} \exp (-\mathrm{j} \Delta \Phi) E_{\mathrm{iv}} \boldsymbol{e}_{\mathrm{v}} \\
& =E_{\mathrm{iu}} \boldsymbol{e}_{\mathrm{u}}+\exp \left(-\mathrm{j} 180^{\circ}\right) E_{\mathrm{iv}} \boldsymbol{e}_{\mathrm{v}} \\
& =E_{\mathrm{iu}} \boldsymbol{e}_{\mathrm{u}}-E_{\mathrm{iv}} \boldsymbol{e}_{\mathrm{v}}=E_{\mathrm{y}} \boldsymbol{e}_{\mathrm{x}} .
\end{aligned}
$$

Thus, a dual-band polarization rotation metasurface is achieved.

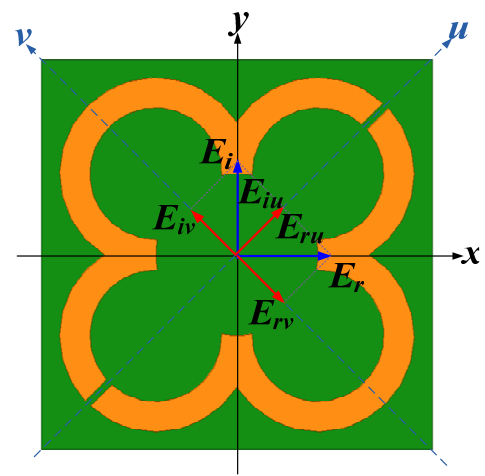

Fig. 5. Intuitive image of y-to-x polarization conversion.

\section{Fabrication and Measurement}

To verify our simulation results, the proposed PRMS is fabricated using a combination of $18 \times 18$ unit cells with an area of $187.2 \mathrm{~mm} \times 187.2 \mathrm{~mm}$, shown in Fig. 7(a). Agilent N5230C vector network analyzer, connected with two standard linearly-polarized horn antennas, is used to

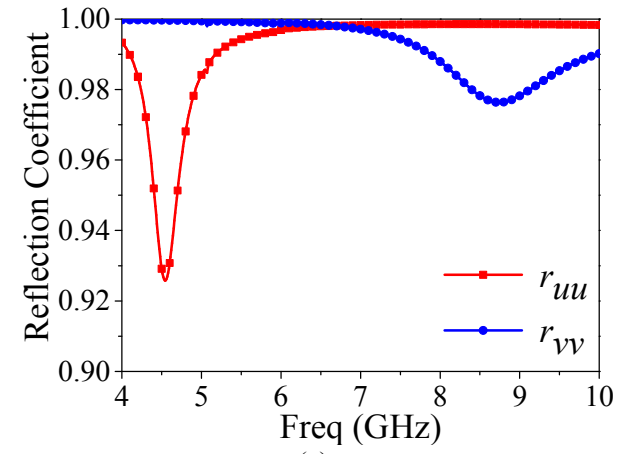

(a)

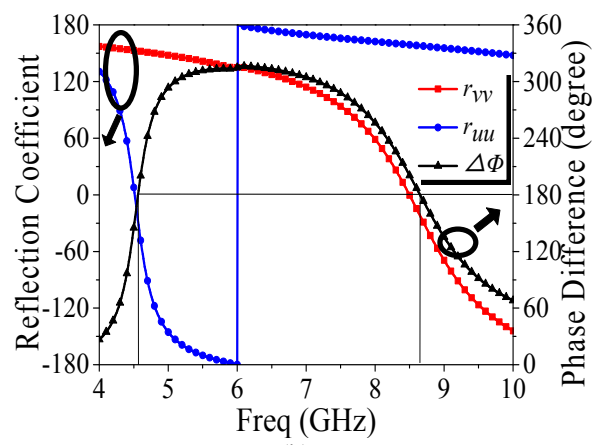

(b)

Fig. 6. The reflection coefficient of $r_{\mathrm{uu}}$ and $r_{\mathrm{vv}}$ : (a) The magnitude; (b) The phase and phase difference.

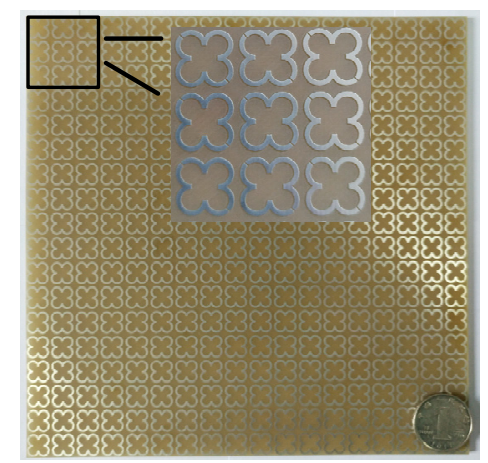

(a)

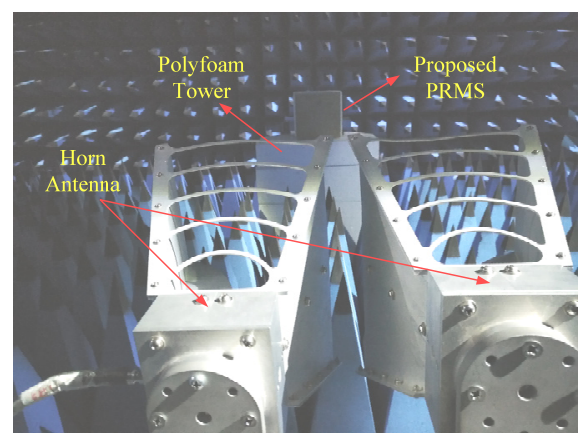

(b)

Fig. 7. The photograph of the proposed PRMS: (a) The fabrication; (b) The experimental setup.

measure the reflection coefficient of the proposed PRMS in an EM anechoic chamber, depicted in Fig. 7(b). The sample was placed at a distance of $150 \mathrm{~cm}$ away from the horn antennas. Moreover, the undesirable signals are eliminated 


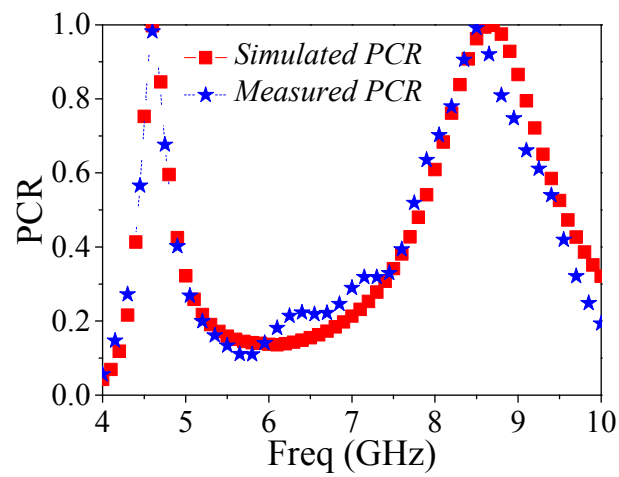

Fig. 8. The measured PCR of the proposed PRMS.

by the time-domain gating. To measure the reflected copolarization and cross-polarization wave, the receiving antenna needs to be rotated $0^{\circ}$ and $90^{\circ}$.

The measured results of the reflection coefficient and PCR are shown in Fig. 8. The measurement results show that the proposed PRMS can convert the linearly-polarized wave to its orthogonal component at $4.60 \mathrm{GHz}$ and $8.50 \mathrm{GHz}$ with high polarization rotation efficiency. The measurement results are in good agreement with the simulation results. The small difference between measurement results and simulation ones might be due to: 1 . Some manufacturing error exists in the fabrication; 2 . The dimension of PRMS is infinite in the simulation, while the dimension of the fabricated PRMS is finite resulting in the edge diffraction; 3 . The incident wave is slightly oblique in the measurements, while the incident wave is under normal direction in the simulation.

\section{Conclusion}

In this paper, a $\mathrm{C} / \mathrm{X}$ dual-band wide-angle reflective PRMS is demonstrated, with numerical simulation, theoretical analysis and experimental measurement. The proposed PRMS realizes nearly $100 \%$ polarization rotation efficiency at $4.61 \mathrm{GHz}$ and $8.67 \mathrm{GHz}$, at which the linear polarization incident wave can be converted into its orthogonal polarization after reflection. Besides, the PRMS can remain high polarization rotation efficiency under an oblique incident direction from $0^{\circ}$ to $60^{\circ}$. The measurement results are in good agreement with the simulation ones. The proposed PRMS has great potential to realize dual-band polarization rotation, radar section cross reduction, and so on.

\section{Acknowledgments}

This work is supported by the National Natural Science Foundation of China (No. 61271100, No. 61471389, No. 61671464 and No. 61501494). Authors also thank reviewers for their valuable comments.

\section{References}

[1] CHEN, P. Y., ALU A. Mantle cloaking using thin patterned metasurfaces. Physical Review B - Condensed Matter and Materials Physics, 2011, vol. 84, no. 20, p. 205110-(1-13). DOI: 10.1103/PhysRevB.84.205110

[2] WANG, J., QU, S.B., XU, Z., et al. Super-thin cloaks mediated by spoof surface plasmons. Photonics and Nanostructures: Fundamentals and Applications, 2012, vol. 10, no. 4, p. 540-546. DOI: 10.1016/j.photonics.2012.04.007

[3] LEE, J., YOO, M., LIM, S. A study of ultra-thin single layer frequency selective surface microwave absorbers with three different bandwidths using double resonance. IEEE Transactions on Antennas and Propagation, 2015, vol. 63, no. 1, p. 221-230. DOI: 10.1109/TAP.2014.2365826.

[4] LI, S., GAO, J., CAO, X., et al. Wideband, thin, and polarizationinsensitive perfect absorber based the double octagonal rings metamaterials and lumped resistances. Journal of Applied Physics, 2014, vol. 116, p. 043710-(1-6). DOI: 10.1063/1.4891716

[5] LEE, D., HWANG, J. G., LIM, D., et al. Incident angle- and polarization-insensitive metamaterial absorber using circular sectors. Scientific Reports, 2016, vol. 6, article no. 27155. DOI: $10.1038 /$ srep 27155

[6] SHI, H., LI, J., ZHANG, A., et al. Gradient metasurface with both polarization-controlled directional surface wave coupling and anomalous reflection. IEEE Antennas and Wireless Propagation Letters, 2015, vol. 14, p. 104-107. DOI: 10.1109/LAWP.2014.2356483

[7] MA, H. F., WANG, G. Z., KONG, G. S., et al. Independent controls of differently-polarized reflected waves by anisotropic metasurfaces. Scientific Reports, 2015, vol. 5, article no. 9605. DOI: $10.1038 /$ srep09605

[8] WANG, J., SHEN, Z., WU, W. Cavity-based high-efficiency and wideband $90^{\circ}$ polarization rotator. Applied Physics Letters, 2016, vol. 109 , no. 15 , p. $153504-(1-5)$. DOI: $10.1063 / 1.4964855$

[9] LI, Y., ZHANG, J., QU, S., et al. Achieving wide-band linear-tocircular polarization conversion using ultra-thin bilayered metasurfaces. Journal of Applied Physics, 2015, vol. 117, p. 044501-(1-7). DOI: 10.1063/1.4906220

[10] YE, Y., HE, S. $90^{\circ}$ polarization rotator using a bilayered chiral metamaterial with giant optical activity. Applied Physics Letters, 2010, vol. 96, no. 20, p. 203501-(1-3). DOI: 10.1063/1.3429683

[11] SONG, K., ZHAO, X., LIU, Y., et al. A frequency-tunable $90^{\circ}$ polarization rotation device using composite chiral metamaterials. Applied Physics Letters, 2013, vol. 103, no. 10, p. 101908-(1-4). DOI: $10.1063 / 1.4820810$

[12] ZHANG, W., POTTS, A., PAPAKOSTAS, A., et al. Intensity modulation and polarization rotation of visible light by dielectric planar chiral metamaterials. Applied Physics Letters, 2005, vol. 86, no. 23, p. 231905-(1-3). DOI: 10.1063/1.1944211

[13] YANG, W., TAM, K., CHOI, W., et al. Polarisation rotation reflective surface based on artificial magnetic conductor and its application. Electronic Letters, 2014, vol. 50, no. 21, p. 1500-1502. DOI: $10.1049 / \mathrm{el} .2014 .2367$

[14] JIA, Y., LIU, Y., ZHANG, W., et al. Ultra-wideband and highefficiency polarization rotator based on metasurface. Applied Physics Letters, 2016, vol. 109, no. 5, p. 051901-(1-4). DOI: $10.1063 / 1.4960355$

[15] LI, S. J., CAO, X. Y., XU, L. M., et al. Ultra-broadband reflective metamaterial with RCS reduction based on polarization convertor, information entropy theory and genetic optimization algorithm. 
Scientific Reports, 2016, vol. 6, article no. 37409. DOI: 10.1038/srep37409

[16] HUANG, X., YANG, D., YANG, H. Multiple-band reflective polarization converter using U-shaped metamaterial. Journal of Applied Physics, 2014, vol. 115, no. 10, p. 103505-(1-6). DOI: $10.1063 / 1.4868076$

[17] SU, P., ZHAO, Y., JIA, S., et al. An ultra-wideband and polarization-independent metasurface for RCS reduction. Scientific Reports, 2016, vol. 6, article no. 20387. DOI: 10.1038/srep20387

[18] SHI, H., ZHANG, A., ZHENG, S., et al. Dual-band polarization angle independent $90^{\circ}$ polarization rotator using twisted electricfield-coupled resonators. Applied Physics Letters, 2014, vol. 104, no. 3, p. 034102-(1-4). DOI: 10.1063/1.4863227

[19] ZHANG, Z., CAO, X., GAO, J., et al. Broadband metamaterial reflectors for polarization manipulation based on cross/ring resonators. Radio Engineering, 2016, vol. 25, no. 3, p. 436-441. DOI: $10.13164 /$ re.2016.0436

[20] HUANG, Y., YANG, L., LI, J., et al. Polarization conversion of metasurface for the application of wide band low-profile circular polarization slot antenna. Applied Physics Letters, 2016, vol. 109, no. 5, p. 054101-(1-5). DOI: 10.1063/1.4960198

[21] ZHANG, L., ZHOU, P., LU, H., et al. Ultrathin reflective metamaterial polarization rotator based on multiple plasmon resonances. IEEE Antennas and Wireless Propagation Letters, 2015, vol. 14, p. 1157-1160. DOI:10.1109/LAWP.2015.2393376

[22] JIA, Y., LIU, Y., GONG, S. Wideband high-gain circular polarized planar antenna based on polarization rotator. In 2016 International Conference on Electromagnetics in Advanced Applications (ICEAA). Cairns (Australia), 2016, p. 416-419. DOI: 10.1109/ICEAA.2016.7731415

[23] LIU, N., GIESSEN, H. Coupling effects in optical metamaterials. Angewandte Chemie International Edition, 2010, vol. 49, no. 51, p. 9838-9852. DOI: 10.1002/anie.200906211

\section{About the Authors ...}

Yulong ZHOU was born in Xian, Shaanxi province, P.R. China in 1993. He received B.Sc. from the Air Force Engineering University, Xi' an China, in 2015. He is currently working toward M.Sc. degree at the Information and Navigation College, Air Force Engineering University. His main interests include wideband antennas, metasurface design and RCS reduction. He is awarded the 2016 Second Prize of the National Graduate Mathematical Modeling Contest.
Xiangyu CAO received the B.Sc and M.A.Sc. degrees from the Air Force Missile Institute in 1986 and 1989, respectively. She received Ph.D. degree in Missile Institute of Air Force Engineering University in 1999. From 1999 to 2002, she was engaged in postdoctoral research in Xidian University, China. She was a Senior Research Associate in the Dept. of Electronic Engineering, City University of Hong Kong from June 2002 to Dec 2003. She is currently a professor of Information and Navigation College of the Air Force Engineering University of CPLA. She is the IEEE senior member from 2008. She has authored and coauthored more than 200 technical journal articles and conference papers, and holds one China soft patent. Her research interests include smart antennas, electromagnetic metamaterial and their antenna applications, and electromagnetic compatibility. She is a reviewer of Applied Physics Letter, Journal of Applied Physics, IEEE Transactions on Antennas \& Propagation, and IEEE Antennas Wireless Propagation Letter.

Jun GAO received the B.Sc and M.A.Sc degrees from the Air Force Missile Institute in 1984 and 1987, respectively. He joined the Air Force Missile Institute in 1987 as an assistant teacher. He became an associate professor in 2000. He is currently a professor of Information and Navigation College, Air Force Engineering University of CPLA. He has authored and coauthored more than 100 technical journal articles and conference papers, and holds one China soft patent. His research interests include smart antennas, electromagnetic metamaterial and their antenna applications.

Sijia LI has received the M. Eng. degree and Ph.D. degree in Electronic Science and Technology (Electronic Field and Microwave Technology) at the Information and Navigation Institute of Air Force Engineering University, Xi'an China, in 2011 and 2015 respectively. He is currently working as an instructor in the Information and Navigation Institute, Air Force Engineering University. His research activity has been focused in the broadband perfect metamaterial absorber, polarization rotation metasurface, and their application for RCS reduction of antennas. He has authored and coauthored more than 40 scientific papers in major journals and international conferences. 